\title{
Effect of plasma treatment on Lyocell fabric/PLA
}

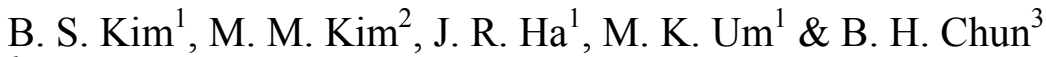 \\ ${ }^{1}$ Composite Materials Lab, Korea Institute of Materials Science, \\ South Korea \\ ${ }^{2}$ Department of Mechanical, Robotics and Energy Engineering, \\ Dongguk U., South Korea \\ ${ }^{3}$ Nano Bio Tech, South Korea
}

\begin{abstract}
Atmospheric glow discharge (AGD) was developed with industrial plasma sources by using high voltage radio frequency (RF) excitation at $\mathrm{KHz}$ frequency ranges. It is possible to produce a steady-state uniform glow discharge at atmospheric pressure with various gases, thus eliminating the requirement of a vacuum system to expose the materials to plasma. The RF frequency should be set in the limited range to produce AGD: if it is too low, the discharge will not initiate and if it is too high, the plasma will either form a filamentary discharge between the electrode plates or it will be transformed to arc discharge. The advantage of AGD is to modify or coat the materials with good uniformity and making use of the AGD surface modification process, a strong interfacial adhesion can be formed between the constituents and the matrix material. Hence, AGD polymerization may be used to modify the surface properties of Lyocell fabric (Acetate Taffeta) and Poly Lactic Acid (PLA) matrix.

In the AGD process, Helium is used as a carrier gas and the monomer (Acrylic Acid) is used to modify the fibre surface property by plasma polymerization. The reactor is operated at room temperature while the surface is modified at atmospheric pressure.

Peel and tensile strength test, and characterizations by SEM were carried out for Lyocell fabric/PLA. Compared to raw fabric, plasma and Silane treatments have improved the tensile strength at a maximum of 30.4 and $27.4 \%$, respectively.
\end{abstract}

Keywords: Lyocell, Poly Lactic Acid, atmospheric glow discharge, Silane. 


\section{Introduction}

With the recent emphasis on materials and processes which are environmentally sustainable, there has been an increased interest in the development of biocomposites based on natural fibres. Besides being derived from renewable sources, the natural fibres suitable for composites are also biodegradable and their production consumes on average 60 percent less energy than the manufacturing of the glass fibres [1].

Cellulose originating from wood or annual plants has been studied as reinforcement in both thermoplastic and thermoset composites for several years [2-6]. However, fabricating cellulose fibre with PLA often leads to poor mechanical properties of composites. The poor mechanical properties of cellulose fibre reinforced PLA composites are due to poor compatibility between the polar hydrophilic fibre and the non-polar hydrophobic PLA, which has weak interfacial adhesion between fibre and PLA matrix.

The objective of this study is to introduce a new environmental friendly method of processing Lyocell fabric/PLA composite using plasma treatment on the Lyocell fabric. This method makes use of non toxic chemical bonding agents. The fabric was first vacuum dried before plasma treated in a continuous process at an atmospheric pressure and room temperature without vacuum. The sizing on the received fabric was done with a solution containing Poly Vinyl Alcohol (PVA), acryl and water at the ratio of 3:1:96. All tests were then performed without removing the sizing.

\section{Atmospheric glow discharge}

\subsection{Selection of monomer}

Acrylic acid or allylamine may be used as precursors to create a hydrophilic fabric surface [6]. The $\pi$ bond of the double bonds in acrylic acid and allylamine has lower bond energy than the others. Hence, the bonds can be broken easily by an electron in plasma state. After which, it becomes radicals with carboxyl group $(-\mathrm{COOH})$ or with amine group $\left(-\mathrm{NH}_{2}\right)$, and they can be grafted on the fibre surface. These carboxyl and amine groups are hydrophilic. Acrylic acid, an oxygen containing compound, is able to produce - $\mathrm{CO}-,-\mathrm{OCO}-,-\mathrm{OH},-\mathrm{O}-$ chains and groups on the fibre surface in plasma state. Those chains and functional groups can also make the surface energy of the fibre higher and hydrophilic. Thus, acrylic acid was selected as the monomer for plasma polymerization.

\subsection{Plasma polymerization}

As shown in Figure 1, the Lyocell fabric is allowed to pass between the electrode plates where the plasma polymerization is formed. Helium is used as a carrier gas and acrylic acid is used to modify the surface property by plasma polymerization. The frequency and voltage used were $20 \mathrm{KHz}$ and $3 \mathrm{KV}$, respectively while the fabric's exposure time to plasma was varied. 


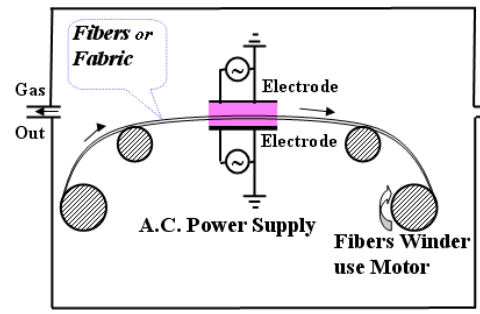

Plasma Chamber
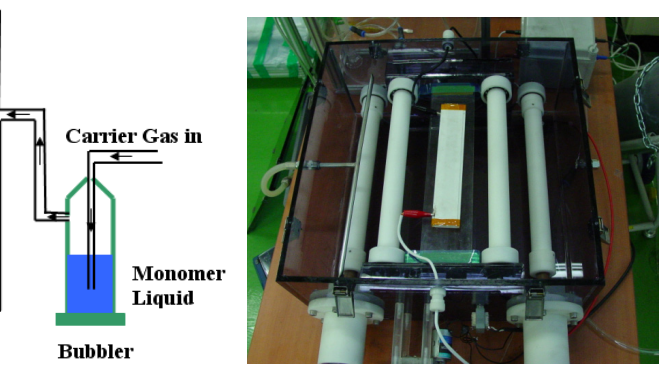

Figure 1: Continuous process of surface coating of Lyocell fabric.

\section{Preparation of the specimen}

\subsection{Materials}

Lyocell fabric:

Acetate Taffeta, SK Chemical, Number of yarns in both fill and warp is $42 / \mathrm{cm}$.

Fibre diameter is $11 \mu \mathrm{m}$

Tensile strength of mono filament is $0.5-0.8 \mathrm{Gpa}$

Poly lactic acid:

GC8000R, Green Chemical, Density 1.042 g/cc, Melting point $150^{\circ} \mathrm{C}$

\subsection{Silane treatments}

Three types of Silane coupling agent concentrations at $0 \sim 5 \%$ were used, respectively, to modify the surface property of Lyocell fabric (Actate Taffeta) in an attempt to improve the interfacial bonding with Poly Lactic Acid (PLA). The fabrics were immersed in a solution of Silane concentration individually for 10 minutes before washed with distilled water for $25 \mathrm{mins}$ and dried in an oven. The Silanes used were Aminoethylaminopropyltrimethoxysilane (6020), 3-Glycidoxy -propyltrimethoxysilane (6030) and 3-Methacryloxypropyltrimethoxysilane (6040), respectively. These Silanes were chosen as the -OH groups in Silane always react with the $-\mathrm{OH}$ groups in PLA. In addition, all three Silanes have similar chemical structures with that of Lactic Acid.

\subsection{Fabrication of composites}

Lyocell fabric/PLA plates $\left(200 \times 100 \times 3 \mathrm{~mm}^{3}\right)$ were fabricated in a hot press (Fig. 2) at $180^{\circ} \mathrm{C}$ and $50 \mathrm{Kpa}$ pressure for 50 minutes under vacuum of $10^{-3}$ Torr. 30 plies of fabrics were alternatively stacked up with 31 plies of PLA films in the fabrication. The fibre volume contents for $6020-2 \%, 6020-3 \%, 6030-2 \%, 6030-$ $3 \%, 6040-2 \%$ and $6040-3 \%$ Silane treated specimens are 59.22, 59.08, 59.22, $58.78,58.99$ and $59.07 \mathrm{wt} \%$, respectively. The fibre volume contents for plasma treated specimens for 2 and 3 mins are 59.47 and $59.01 \mathrm{wt} \%$, respectively. 


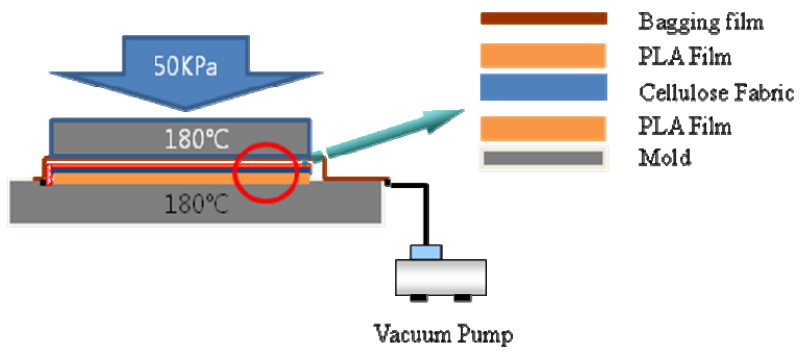

Figure 2: Fabrication on hot press.

\subsection{Peel tests}

Peel tests (Fig. 3) for raw and treated fabrics attached to the PLA by the injection machine (Woojin Selex, Selex-NE55) were carried out, respectively, by peel test device (Jungdo Instrument, DP-1). Each fabric was peeled off at a speed of $50 \mathrm{~mm} / \mathrm{min}$ and the peeled off area was $10 \times 70 \mathrm{~mm}^{2}$. Five specimens were tested for each treatment conditions.

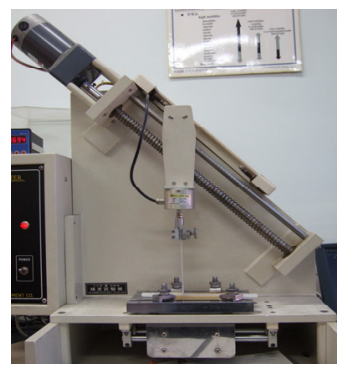

Figure 3: Peel test.

\section{Results and discussion}

Peel test results for the fabrics treated with varying Silane concentrations and plasma treated with different exposure time are shown in Figure 4. The results with varying Silane concentrations, 6030-1\%, 6030-2\%, 6030-3\%, 6040-2\% showed highest and most efficient values for the practical purposes. For the plasma treatment, the exposure to plasma for 270 seconds showed the highest value, the most efficient plasma exposure time appears to be between $60-120$ minutes.

The tensile test specimens were prepared according to ASTM D638 Type V. Figure 5 represents tensile strength of Lyocell fabric/PLA for the fabrics treated with varying Silane concentrations and plasma treated with different exposure time. Comparing it with the raw fabric/PLA, 6030-2\% Silane treatment showed the biggest improvement of $27.4 \%$ while in plasma treatment case, 2 minutes treatment provided similar improvement of $30.4 \%$. 

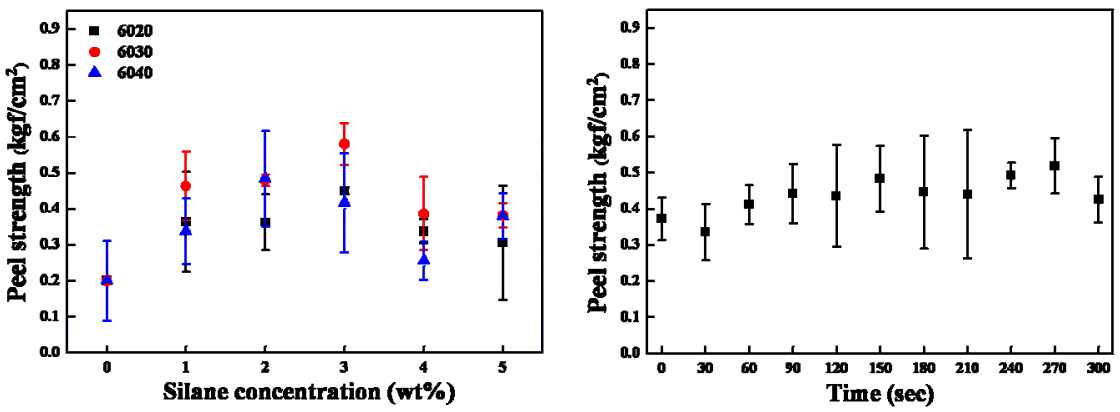

Figure 4: $\quad$ Peel test results.
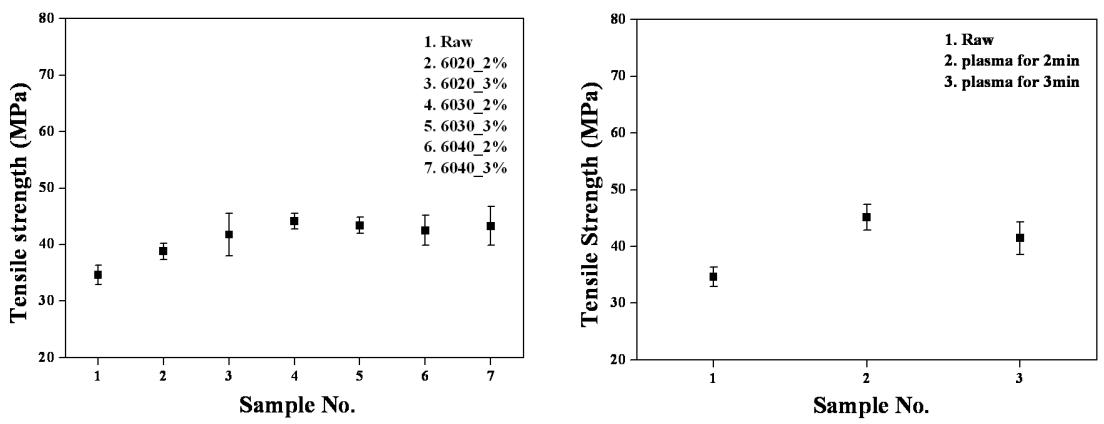

Figure 5: Tensile test results.

Figure 6 represents the tensile test specimens and the change in color was noted. Plasma treated specimen turned light brown and Silane treated specimen turned into a darker color. The reaction between the $\mathrm{OH}$ group of PLA of the sizing material and Silane might be one of the reasons for such change in colors. The $\mathrm{OH}$ group in PLA might have reacted with the $\mathrm{OH}$ on the fabric after plasma treatments to result in color change too. This may be indirectly verified by Fig. 6(d) which is 6030- 2\% Silane treated fabric/PLA with the sizing removed.

\subsection{SEM on Lyocell fabric/PP}

Figure 7 represents the Scanning Electron Microscopic views of the fracture surface of the raw, plasma and Silane treated Lyocell fabric/PLA. Since the fabric reinforced composites is used, the fracture surface is much rougher than unidirectional fibre reinforced composites. The bonding between the fibre and matrix for plasma and Silane composites appears to be better than that of non treated (raw) fabric.

Densities of the composites with raw fabric, Silane treated and plasma treated were $1.285,1.234$ and $1.235 \mathrm{~g} / \mathrm{cm}^{3}$, respectively. 


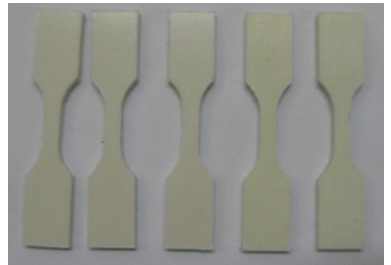

(a) raw

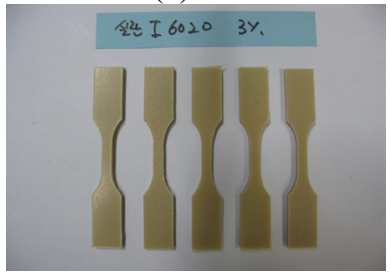

(c) $6020-3 \%$ Silane treated

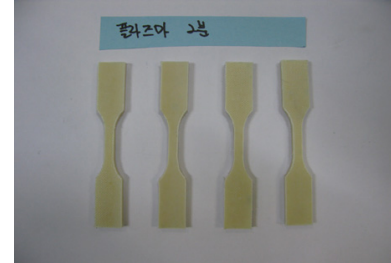

(b) plasma treated, $2 \mathrm{~min}$

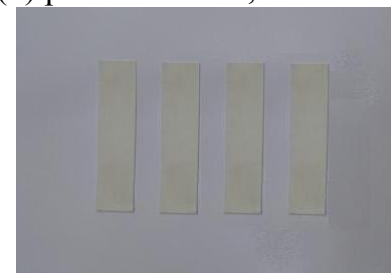

(d) $6020-3 \%$ Silane treated w/o sizing

Figure 6: Lyocell fabric/PLA tensile specimens.
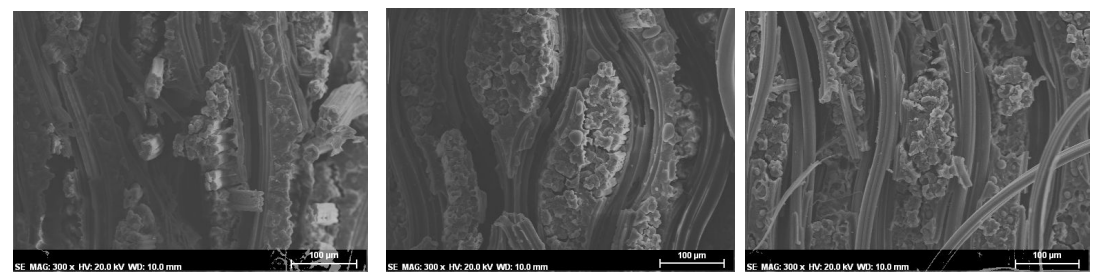

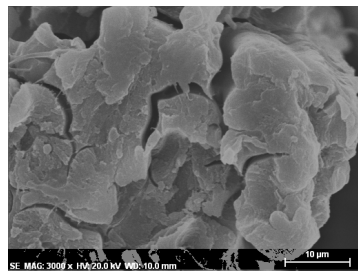

raw

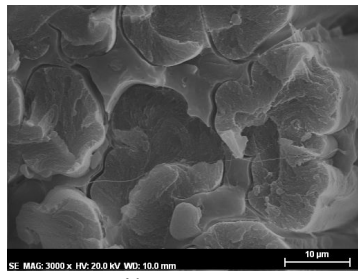

Silane 3\%

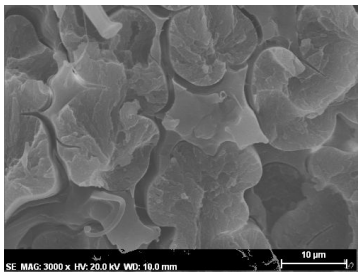

plasma 3 mins

Figure 7: $\quad 50 \mathrm{wt} \%$ of wood powder within PP. The ones in circle represent the wood powder.

\section{Conclusions}

1. In plasma polymerization, Acrylic acid was used as precursors to make the surface of Lyocell fabric hydrophilic. Three types of Silanes were also used to modify the surface property of Lyocell fabric. Compared to raw fabric, plasma and Silane treatments have improved the tensile strength at maximum of 30.4 and $27.4 \%$, respectively.

2. Both plasma and Silane treatment have caused the change in color of the fabricated specimen. This may be due to the reaction between the $\mathrm{OH}$ 
group in PLA of the sizing materials and the $\mathrm{OH}$ group in Silane and the plasma treated fabric.

\section{Acknowledgement}

Present research was performed as an International Collaboration Project supported by the Ministry of Knowledge Economy (MKE).

\section{References}

[1] Brosius D, Natural Fiber Composites slowly take root, Comp Technol 2006; 32-37.

[2] Gellerstedt F, Gatenholm P, Surface properties of lignocellulosic fibers bearing carboxylic group, Cellulose 1999; 6(2):103-121

[3] Ganster J, Fink H P, Novel cellulose fiber reinforced thermoplastic materials, Cellulose 2006; 13(3):271-280

[4] Laka M, Chernyavskaya S, Maskavs M, Cellulose-Containing Fillers for Polymer Composites, Mechanics of Composite Materials 2003;39(2):183188

[5] Orts W J et al, Application of Cellulose Microfibrils in Polymer Nanocomposites, J Polym and the Environment 2005;13(4):301-306

[6] H. Yasuda, Plasma Polymerization, Academic Press, Orlando, Florida, 1985 